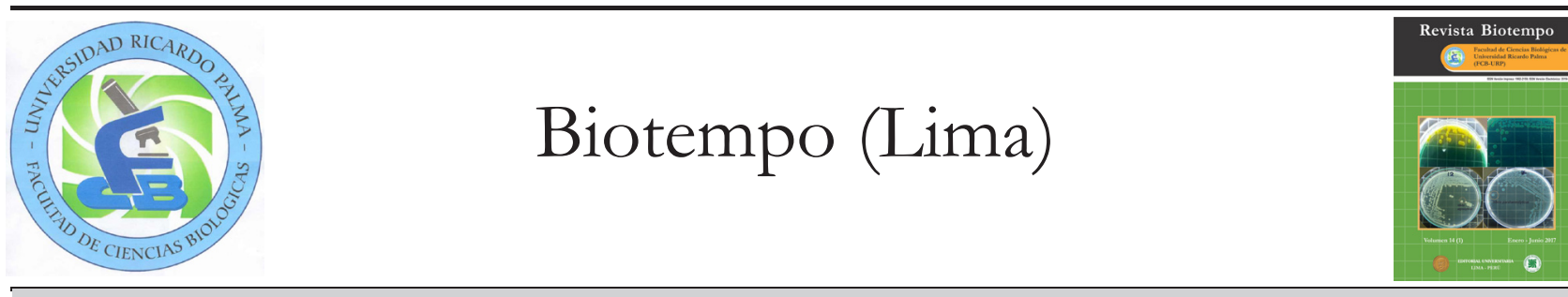

ORIGINAL ARTICLE/ ARTÍCULO ORIGINAL

ISOLATION AND IDENTIFICATION OF BACTERIA OF THE GENUS VIBRIO ON SAMPLES OF AULACOMYA ATRA “CHORO” FROM THE FISHING TERMINAL OF VILLA MARIA DEL TRIUNFO, LIMA, PERU

\title{
AISLAMIENTO E IDENTIFICACIÓN DE BACTERIAS DEL GENERO VIBRIO EN MUESTRAS DE AULACOMYA ATRA "CHORO" PROCEDENTES DEL TERMINAL PESQUERO DE VILLA MARÍA DEL TRIUNFO, LIMA, PERÚ
}

\section{Bryan Canal'; Sergio Cruz'; Valeria Valle-Riestra ${ }^{1}$; Juan Carlos Ramos ${ }^{2} \&$ Tomas Agurto $^{2}$}

\footnotetext{
${ }^{1}$ Universidad Ricardo Palma, Facultad de Ciencias Biológicas. ${ }^{2}$ Instituto de Control y Certificación de la Calidad e Inocuidad Alimentaria de la Universidad Ricardo Palma - ICCCIA-URP.

carlos.ramosg@urp.pe / icccia.urp@urp.edu.pe
}

\section{ABSTRACT}

\begin{abstract}
Bivalve molluscs are considered organisms of great economic and social importance. However, they have been identified as agents of food poisoning. Therefore, the objective of this work was to evaluate the isolation and identification of bacteria of the genus Vibrio in samples of Aulacomya atra "choro" from the fishing terminal of Villa María del Triunfo, Lima, Peru, which link them with outbreaks of enteric conditions. A total of 30 samples of the bivalve mollusc $A$. atra was analyzed. Interest was focused on species of the genus Vibrio, using the methodology recommended by the Food and Drug Administration (FDA) and Bacteriological Analytical Manual (BAM) for the isolation of Vibrio in samples of marine origin. Of the 30 analyzed samples, a total of 8 Vibrio strains was isolated, predominantly Vibrio alginolyticus. No Vibrio cholerae was present. Seven strains corresponded to Vibrio alginolyticus and 1 strain to Vibrio parahaemolyticus, responsible for gastroenteritis due to the consumption of raw or undercooked seafood.
\end{abstract}

Keywords: Molluscs - Vibrio cholera - Foodborne diseases

\section{RESUMEN}

Los moluscos bivalvos son considerados organismos de gran importancia económica y social. Sin embargo, han sido identificados como agentes de toxiinfecciones alimentarias. En consecuencia, el objetivo de este trabajo fue evaluar aislar e identificar bacterias del genero Vibrio en muestras de Aulacomya atra "choro" procedentes del terminal pesquero de Villa María del Triunfo, Lima, Perú, que los vinculan con brotes de afecciones entéricas. Se analizaron un total de 30 muestras del molusco bivalvo $A$. atra. Se centró el interés por especies del género Vibrio, utilizándose la metodología recomendada por la "Food and Drug Administration" (FDA) y "Bacteriological Analytical Manual” (BAM) para el aislamiento de Vibrio en muestras de origen marino. De las 30 muestras analizadas se aislaron un total de 8 cepas de Vibrio, con predominancia Vibrio alginolyticus. No hubo presencia de Vibrio cholerae. Siete cepas correspondieron a Vibrio alginolyticus y 1 cepa a Vibrio parahaemolyticus, responsable de la gastroenteritis por el consumo de mariscos crudos o poco cocidos.

Palabras claves: Moluscos - Vibrio cholerae - Toxiinfecciones alimentarias 


\section{INTRODUCCIÓN}

El género Vibrio, es una bacteria Gram negativas cuyo hábitat son los ambientes marinos y estuarinos. Sin embargo, algunas especies de Vibrio son patógenos en humanos, siendo Vibrio parahaemolyticus, Vibrio vulnificus y Vibrio cholerae los más importantes. En este sentido, $V$. parahaemolyticus es reconocido en todo el mundo como el agente causal de la gastroenteritis humana resultante del consumo de mariscos crudos o poco cocidos. $V$. vulnificus ha sido frecuentemente asociado a infecciones graves causadas por la exposición de las heridas al agua de mar. Finalmente, $V$. cholerae es el agente causal del cólera y es de relevancia especial, ya que se ha detectado en aguas salobres en todo el mundo (Tall et al., 2012; Cantet et al., 2013); Mannas et al., 2014.

En los Estados Unidos, V. vulnificus es responsable del 95 por ciento de todas las muertes después de la ingestión de mariscos crudos o poco cocidos. Diferentes factores han sido implicados en la virulencia de $V$. vulnificus, Incluyendo el gen vvhA que codifica la Citolisina hemolítica (Oliver, 2006). Vibrio cholerae, el agente causal de cólera, se ha detectado en aguas naturales y salobres en todo el mundo. Esta especie también ha sido aislada de áreas donde no se han reportado casos clínicos de cólera (Colwell et al., 1977). Sin embargo, la mayoría de los aislamientos medioambientales son de $V$. cholerae no O1/no O139 capaz de causar brotes de diarrea a nivel local (Rippey, 1994).

La ocurrencia de brotes de infección debido a cepas de vibrios ambientales no se ha considerado de relevancia clínica, pero se ha correlacionado con los factores de virulencia (González-Escalona et al., 2006). Debido a estos problemas, un monitoreo rápido y confiable de las cepas patógenas de Vibrio y sus factores de virulencia en los productos marinos o hidrobiológicos son de gran interés para la salud humana y la economía. Los métodos de cultivo estándar de laboratorio utilizados para identificar los Vibrios patógenos tienen estas características: consumen mucho tiempo, requieren mucha mano de obra, no atacan los factores de virulencia asociados con enfermedades humanas $y$ no pueden detectar los microorganismos en el estado viable, pero no cultivable (Vora et al., 2005).

La necesidad de vigilar adecuadamente las amenazas biológicas en salud ha impulsado el desarrollo de tecnologías avanzadas investigación para la detección de patógenos. Los métodos moleculares son adecuados i) para una detección independiente del cultivo del microorganismos, ii) identificar con precisión las especies bacterianas, y iii) proporcionar datos sobre el potencial patogénico de los microorganismos (Wilson et al., 2002).

En consecuencia, el objetivo de este trabajo fue aislar e identificar bacterias del género Vibrio en muestras de Aulacomya atra (Molina, 1782) "choro" procedentes del terminal pesquero de Villa María del Triunfo, Lima, Perú.

\section{MATERIAL Y MÉTODOS}

Obtención y procesamiento de la muestra

Fueron colectadas 30 muestras del molusco bivalvo $(A$. atra) "choro" procedentes del Terminal Pesquero de Villa María del Triunfo (12¹0’24.2”S 7656'52.0”W), ubicado entre las Avenidas Pachacutec y María Parado de Bellido en el distrito de Villa María del Triunfo de la Ciudad de Lima, Perú. Se colocaron en bolsas plásticas de cierres herméticos y se transportaron en un cooler con hielo $\left(4^{\circ} \mathrm{C}\right)$ al Laboratorio de Microbiología de la Facultad de Ciencias Biológicas de la Universidad Ricardo Palma, Lima, Perú. Los individuos fueron desvalvados y homogenizados individualmente en una licuadora con solución salina fosfatada suplementada con $2 \%$ de $\mathrm{NaCl}$ durante 4 seg. Luego, se tomó 1 $\mathrm{mL}$ del homogenizado y se inoculó en $9 \mathrm{~mL}$ de agua peptonada alcalina (APA) más $2 \%$ de cloruro de sodio (NaCl). Los tubos se incubaron a $35-37^{\circ} \mathrm{C}$ durante 18 h (FDA/BAM, 1998).

\section{Aislamiento e identificación}

Los tubos con crecimiento positivo, fueron sembrados en agar TCBS. Las placas fueron incubadas a $35-37^{\circ} \mathrm{C}$ por $24 \mathrm{~h}$. Las colonias que presentaron características típicas de Vibrio, fueron seleccionadas para formar parte del cepario, además fueron sembradas en el medio HiCrome Vibrio Agar.

\section{Preparación del cepario y pruebas bioquímicas}

Las colonias seleccionadas fueron sembradas en tubos con agar nutritivo suplementado con $\mathrm{NaCl}$ al: $0 \%, 1 \%$, $6 \%$ y $9 \%$ para la obtención del cepario. Posteriormente, 
se realizó la coloración diferencial de Gram, siembras en medio HiCrome Vibrio Agar y análisis de ensayo bioquímicos en LIA, SIM, Citrato y TSI, así como pruebas de oxidasa y catalasa (FDA/BAM, 1998).

\section{Serología y análisis moleculares}

Se realizaron las pruebas serológicas para los serotipos de $V$. cholerae: O139, Inaba y Ogawa (FDA/BAM, 1998). Además, se realizaron las pruebas con PCR convencional para los genes de virulencia ompW y ctx (Menezes et al., 2014). En colaboración con la Naval Medical Research Unit Six (NAMRU-6), se realizaron las pruebas de tipo Phoenix 100 y PCR convencional.

\section{RESULTADOS}

En la Figura 1 se observa en la parte superior la siembra en agar selectivo y diferencial TCBS, observándose colonias amarillas (Vibrio alginolyticus) y colonias verdes (Vibrio parabaemolitycus), y en la parte inferior se observan las pruebas en medio HiCrome Vibrio Agar. En la Figura 2 se observa la coloración diferencial de Gram realizada a los cepario observándose a los bacilos curvos Gram negativos, confirmándose la presencia del género Vibrio. Los resultados fueron negativos para los ensayos serológicos con el reactivo para O139, Inaba y Ogawa. Se confirma mediante el ensayo de pruebas moleculares que no existe la presencia de Vibrio cholerae para las muestras procesadas (Fig. 3). Pero mediante el equipo automatizado Phoenix 100 se confirmó la presencia de siete cepas de $V$. alginolyticus y una cepa de $V$. parahaemolitycus.

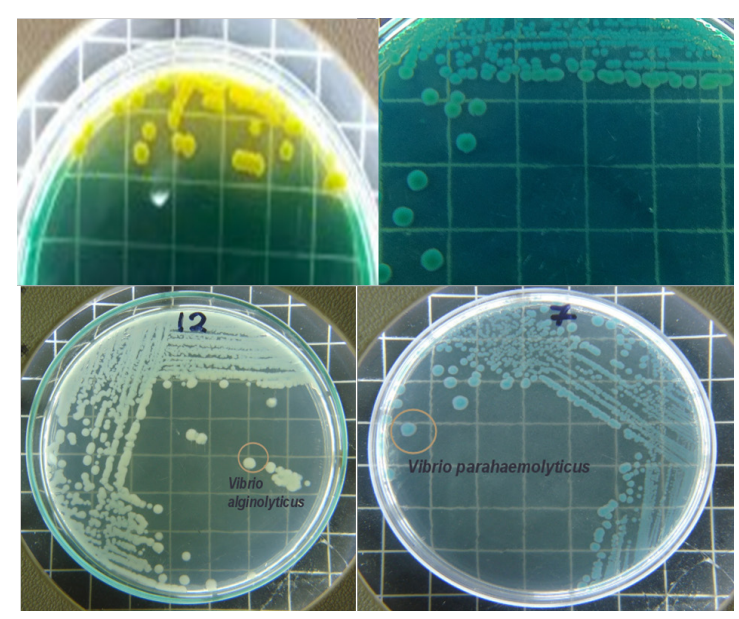

Figura 1. Superior: colonias aisladas en agar TCBS. Inferior: colonias aisladas en HiCrome agar.

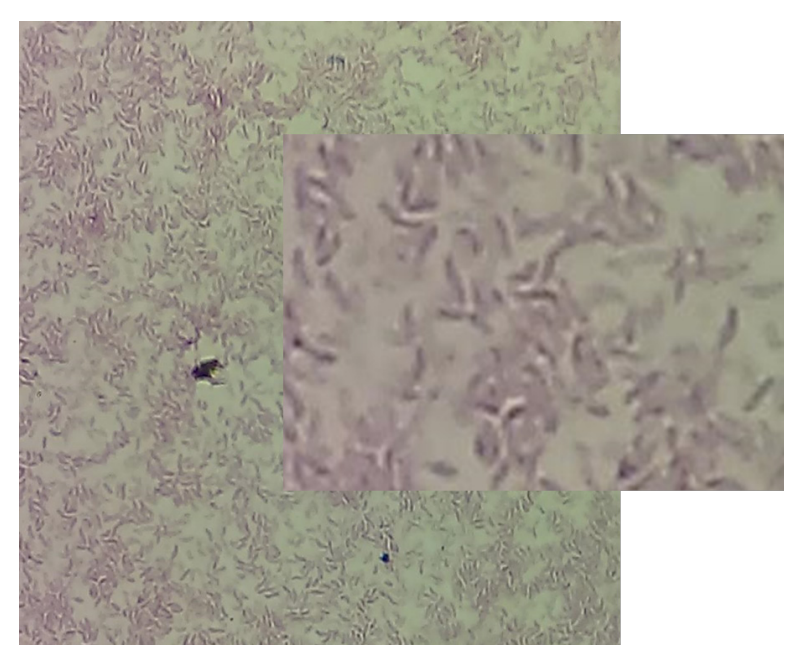

Figura 2. Vibrio: Bacilos curvos Gram negativos.

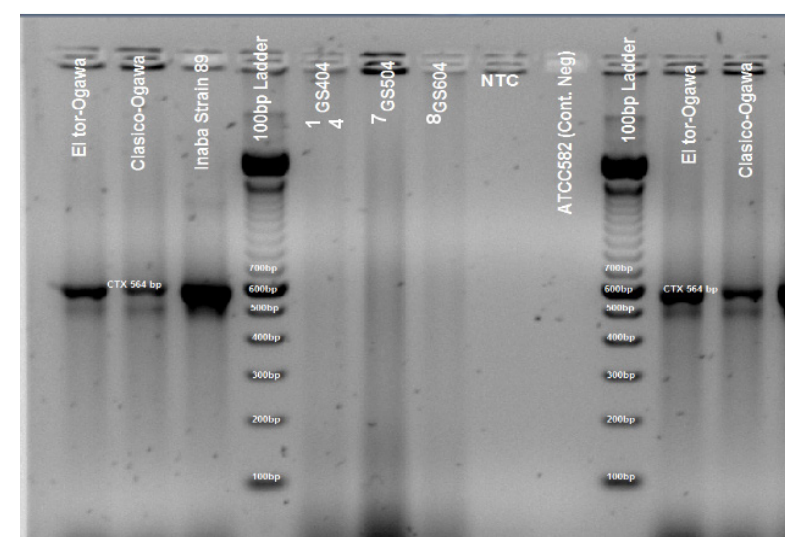

Figura 3. Resultado del perfil electroforético de tres cepas identificadas genotípicamente como Vibrio cholerae utilizando cebadores para el gen específico de la especie OmpW y CTX en PCR.

\section{DISCUSIÓN}

El principal objetivo de este estudio fue el aislamiento e identificación de bacterias del género Vibrio en muestras de $A$. atra "choro" procedentes del Terminal Pesquero de Villa María del Triunfo, Lima, Perú. En comparación con otros estudios que investigaron mejillones (Mytilus edulis y Mytilus galloprovincialis) (Romero et al., 2014), el porcentaje de muestras de $V$ ibrio positivas en el presente estudio fue menor que el encontrado en estudios previos realizados en Alemania (74,4\% en el mar de Wadden), Jónico (Mar Piccolo del Mar de Tarento: 60\%) e Italia (Mar Adriático: 48,4\%) (Ripabelli et al., 1999; Cavallo \& Stabili, 2002; Lhafi \& Kuhne, 2007). La diferencia obtenida entre nuestros 
resultados y estos informes puede estar asociada con la existencia de diferentes condiciones climáticas en las diferentes áreas geográficas.

El uso de dos medios de agar (TCBS y el medio HiCrome Vibrio Agar) en el presente estudio para aislar Vibrio spp., puede influir en la detección del número de muestras positivas con respecto a estudios previos (por ejemplo, Ripabelli et al. (1999) y Lhafi \& Kuhne (2007)) que utilizaron solo medio con TCBS. El medio HiCrome Vibrio Agar contiene sustratos para la beta-galactosidasa. Se desarrolló específicamente para diferenciar $V$. parahaemolyticus, $V$. vulnificus, $V$. cholerae non $\mathrm{O} 1$ non $\mathrm{O} 139$ y otros Vibrio directamente en la etapa de aislamiento en base al color de la colonia y utiliza un sustrato cromogénico (en lugar de la fermentación del azúcar en medio de cultivo tradicional tal como TCBS). En el medio HiCrome Vibrio Agar, las colonias de $V$. parahaemolyticus fueron verde azuladas. Las de $V$. vulnificus y $V$. cholerae parecían purpuras, mientras que las colonias de $V$. alginolyticus eran incoloras. Nuestros resultados sugieren que el

\section{REFERENCIAS BIBLIOGRÁFICAS}

Blanco-Abad, V.; Ansede-Bermejo, J.; RodríguezCastro, A. \& Martinez-Urtaza, J. 2009. Evaluation of different procedures for the optimized detection of Vibrio parahaemolyticus in mussels and environmental samples. International Journal of Food Microbiology, 129:229-236.

Cantet, F.; Hervio-Heath, D.; Caro, A.; Le Mennec, C.; Monteil, C.; Quemer, C. 2013. Quantification of Vibrio parahaemolyticus, $V$. vulnificus and $V$. cholerae in French Mediterranean coastal lagoons. Research in Microbiology, 164: 867874.

Cavallo, R.A. \& Stabili, L. 2002. Presence of vibrios in seawater and Mytilus galloprovincialis (Lam) from the Mar Piccolo of Taranto (Ionian Sea). Water Research, 36: 3719-3726.

Colwell, R.R.; Kaper, J. \& Joseph, S.W. 1977. Vibrio cholerae, Vibrio parabaemolyticus and other vibrios: occurrence and distribution in Chesapeake Bay. Science, 198: 394-396. medio HiCrome Vibrio Agar fue más eficiente y preciso para la identificación de Vibrio en mejillones que el agar TCBS convencional. Este resultado coincidió con Blanco-Abad et al. (2009) y Di Pinto et al. (2011). Vibrio alginolyticus y $V$. parabaemolyticus fueron las especies encontradas en $A$. atra. Vibrio alginolyticus fue la especie más abundante recuperada de las muestras. De hecho, el agua de mar es el hábitat normal de $V$. alginolyticus y se aísla del agua de mar y de mariscos en muchas partes del mundo.

De las 30 muestras analizadas, $8(27 \%)$ presentaron la presencia del género Vibrio, que se evaluaron en los ensayos microbiológicos y bioquímicos realizados. Así mismo, de estas 8 cepas aisladas, ninguna fue identificada como $V$. cholerae mediante las pruebas moleculares empleadas. Se concluyó mediante el equipo automatizado Phoenix 100 que siete cepas (87,5\%) correspondieron a $V$. alginolyticus y una cepa $(12,5 \%)$ a $V$. parahaemolyticus. En conclusión, los resultados observados en el presente estudio mostraron que Vibrio alginolyticus fué el más abundante entre los Vibrio spp.

Di Pinto, A.; Terio, V.; Novello, L. \& Tantillo, G. 2011. Comparison between thiosulphate-citrate bile salt sucrose (TCBS) agar and CHROMagar Vibrio for isolating Vibrio parahaemolyticus. Food Control, 22:124-127.

FDA/BAM (US Food \& Drug Administration). 1998. BAM: Vibrio Bacteriological Analytical Manual. Chapter G. Vibrio. Kaysner, Ch. A. \& De Paola, A. Jr. 8th Edition. Revision A.

González-Escalona, N.; Blackstone, G.M. \& De Paola, A. 2006. Characterization of a Vibrio alginolyticus strain, isolated from Alaskan oysters, carrying a hemolysin gene similar to the thermostable direct hemolysin-related hemolysin gene (trh) of Vibrio parahaemolyticus. Applied and Environmental Microbiology, 72: 7925-7929.

Lhafi, S.K. \& Kuhne, M. 2007. Occurrence of Vibrio spp. in blue mussels (Mytilus edulis) from the German Wadden Sea. International Journal of Food Microbiology, 116:297-300. 
Mannas, H. FDA/BAM; Mimouni, R.; Chaouqy, N.; Hamadi, F. \& Martínez-Urtaza, J. 2014. Occurrence of Vibrio and Salmonella species in mussels (Mytilus galloprovincialis) collected along the Moroccan Atlantic coast. Springer Plus, 3:265. doi:10.1186/2193-1801-3-265.

Menezes, F.G.R.; Neves, S.S.; Sousa, O.V.; Vila-Nova, C.M.V.M.; Maggioni, R.; Theophilo, G.N.D.; Hofer, E. \& Vieira, R.H.S.F. 2014. Detection of virulence genes in environmental strains of Vibrio cholerae from estuaries in Northeastern Brazil. Revista do Instituto de Medicina Tropical de São Paulo, 56: 427-432.

Oliver, J.D. 2006. Vibrio vulnificus. In: Thompson, F.L.; Austin, B. \& Swings, J. (Eds.). Biology of Vibrios. ASM Press, Washington, DC. pp. 349-366.

Ripabelli, G.; Sammarco, M.L.; Grasso, G.M.; Fanelli, I.; Caprioli, A. \& Luzzi, I. 1999. Occurrence of Vibrio and other pathogenic bacteria in Mytilus galloprovincialis (mussels) harvested from Adriatic Sea, Italy. Food Microbiology, 49:43-48.

Rippey, S.R. 1994. Infectious diseases associated with shellfish consumption. Clinical Microbiology Reviews, 4: 419-425.

Romero, A.; Costa, M. M.; Forn-Cuni, G.; Balseiro, P.; Chamorro, R.; Dios, S.; Figueras, A. \&
Novoa, B. 2014. Occurrence, seasonality and infectivity of Vibrio strains in natural populations of mussels Mytilus galloprovincialis. Diseases of Aquatic Organisms, 108: 149_ 163.

Tall, A.; Teillon, A.; Boisset, C.; Delesmont, R.; Touron-Bodilis, A. \& Hervio-Heath, D. 2012. Real-time PCR optimization to identify environmental Vibrio spp. strains. Journal of Applied Microbiology, 113: 361-372.

Vora, G.J.; Meador, C.E.; Bird, M.M.; Bopp, C.A.; Andreadis, J.D. \& Stenger, D.A. 2005. Microarray-based detection of genetic heterogeneity, antimicrobial resistance, and the viable but nonculturable state in human pathogenic Vibrio spp. Proceedings of the National Academy of Sciences, 102: 19109_ 19114.

Wilson, W.J.; Strout, C.L.; DeSantis, T.Z.; Stilwell, J.L.; Carrano, A.V. \& Andersen, G.L. 2002. Sequence-specific identification of 18 pathogenic microorganisms using microarray technology. Molecular and Cellular Probes, 16: 119-127.

Received November 1, 2016.

Accepted November 30, 2016. 